\section{P2-324 ETHNIC DIFFERENCES IN CARDIOVASCULAR RISK IN CHILDHOOD: COMPARISON OF UK SOUTH ASIAN, AFRICAN- CARIBBEAN AND EUROPEAN CHILDREN}

doi:10.1136/jech.2011.142976k.56

\begin{abstract}
${ }^{1} \mathrm{P}$ Whincup, ${ }^{*} \mathrm{C}$ Nightingale, ${ }^{1} \mathrm{~A}$ Donin, ${ }^{2} \mathrm{~A}$ Rapala, ${ }^{2} \mathrm{D}$ Joysurry, ${ }^{2} \mathrm{M}$ Prescott, ${ }^{2} \mathrm{~A}$ Donald, ${ }^{2} \mathrm{E}$ Ellins, ${ }^{1} \mathrm{C}$ Owen, ${ }^{1} \mathrm{~A}$ Rudnicka, ${ }^{1} \mathrm{D}$ Cook, ${ }^{2} \mathrm{~J}$ Deanfield. ${ }^{1}$ St George's, University of London, London, UK; ${ }^{2}$ Institute of Child Health, London, UK
\end{abstract}

Introduction UK South Asian adults have high risks of coronary heart disease, stroke and type 2 diabetes (T2D) when compared with Europeans; UK African-Caribbeans have high risks of stroke and T2D and low coronary heart disease risks. With growing evidence that cardiovascular disease risks begin before adulthood, we compared risk factor patterns and vascular disease markers in UK children from these ethnic groups.

Methods We conducted a school-based study of cardiovascular risk profiles in 4796 9-10 year-old UK children of South Asian, African-Caribbean and European origin, with substudies of carotid intimal-medial thickness (cIMT) and carotid-femoral pulse wave velocity (cfPWV) in 939 and 631 children respectively.

Results Compared with Europeans, UK South Asian children had higher adiposity, diastolic BP, insulin resistance, HbA1c and triglyceride levels; their HDL-cholesterol was low. cfPWV was slightly higher in South Asians and cIMT similar. In contrast, AfricanCaribbean children had lower adiposity, LDL-cholesterol and triglyceride levels and higher diastolic BP, insulin resistance, $\mathrm{HbA1c}$ and HDL-cholesterol; both cfPWV and cIMT were higher in AfricanCaribbeans. CIMT was positively associated with systolic and diastolic BP, while cfPWV was positively associated with adiposity, diastolic BP and insulin resistance. However, adjustment for these risk factors had little effect on the ethnic differences in cfPWV and cIMT observed.

Conclusion Appreciable ethnic differences both in cardiovascular risk factors and vascular disease markers are apparent in children before puberty, which are substantially consistent with adult cardiovascular disease patterns. There may be important opportunities for cardiovascular disease prevention before adult life in high-risk ethnic minority groups.

\section{P2-325 TRENDS IN CARDIOVASCULAR DISEASE TREATMENT IN THE UK, 1961-2011}

doi:10.1136/jech.2011.142976k.57

K Wickramasinghe, ${ }^{*}$ P Bhatnagar, P Scarborough. BHF Health Promotion Research Group, Department of Public Health, University of Oxford, Oxford, UK

Introduction Cardiovascular disease (CVD) is the leading non communicable disease contributing to more than 17 million deaths annually in the world and in the UK for almost 191000. The knowledge base related to the aetiology and treatment of CVD has expanded over last few decades informing effective prevention and treatment efforts. The aim of this study was to report trends in hospital episodes, surgeries and prescriptions for CVD in the UK over the last 50 years.

Methods Data obtained from several national surveys including prescription cost analysis, National Adult Cardiac Database Report, Hospital In-patient Enquiry and Hospital Episodes Statistics.

Results There were around 70000 estimated inpatient episodes of CHD in England and Wales in 1961, increasing to around 450000 by 2009. In 1962, there were 700 surgeries to treat arteriosclerotic heart disease in England and Wales. By 2009, there were around 25000 coronary artery bypass graft surgeries and over 80000 percutaneous coronary interventions carried out annually. In 2008, around 266 million prescriptions (costing $£ 1.6$ billion) were issued for CVD in
England, five times as many as issued in 1986. Since 1990 the number of prescriptions for antiplatelet and lipid lowering drugs have increased steadily.

Conclusion Improvements in treatment for CVD in the UK have contributed to declines in case fatality and mortality rates. But despite these improvements in treatments, CVD remains the biggest killer. Analysis and interpretation of treatment trends has been restricted by data availability and comparability issues such as changes in surgical procedures, classification of diseases and drugs.

\section{P2-326 COMPARISON OF URBAN DIABETICS WITH OPTIMAL AND SUBOPTIMAL CONTROL}

doi:10.1136/jech.2011.142976k.58

${ }^{1} \mathrm{M}$ Pinidiyapathirage, ${ }^{1} \mathrm{~T}$ Warnakulasuriya, ${ }^{1} \mathrm{~A}$ Kasturiratne, ${ }^{1} \mathrm{U}$ Ranawaka, ${ }^{1} \mathrm{D}$ Gunasekera, ${ }^{1} \mathrm{~N}$ Wijekoon, ${ }^{1} \mathrm{~K}$ Medagoda, ${ }^{1} \mathrm{~S}$ Perera, ${ }^{2} \mathrm{~F}$ Takeuchi, ${ }^{2} \mathrm{~N}$ Kato, ${ }^{1}$ A Wickremasinghe. ${ }^{* 1}$ Faculty of Medicine, University of Kelaniya, Ragama, Sri Lanka; ${ }^{2}$ Department of Gene Diagnostics and Therapeutics, Research Institute, National Center for Global health and Medicine, Tokyo, Japan

Introduction The prevalence of Diabetes Mellitus in Sri Lanka is increasing. We describe the characteristics of patients with optimal and suboptimal control of diabetes among known diabetics in a 35-64-year-old urban population resident in the Ragama Medical Officer of Health (Ragama MOH) area of Sri Lanka.

Methods A cross sectional study was conducted among 2986 randomly selected 35-64 year olds in the Ragama $\mathrm{MOH}$ area from January to September 2007. A detailed history was taken and participants were subjected to a physical examination and assay of fasting blood glucose and HbA1C. A HBA1C <6.5 was taken as evidence of optimal control.

Results There were 474 persons (194 males and 280 females) who gave a past history of diabetes. 9 males and 9 females were not on any treatment. 27 persons ( 9 males and 18 females) were on insulin. Of the 474 diabetics, 113 (48 males and 65 females) had a HbA1c $<6.5$. The average fasting blood glucose of diabetics with optimal control was $120+21 \mathrm{mg} / \mathrm{dl}$. The mean fasting blood glucose level of the 361 subjects with sub optimal control was $190+70 \mathrm{mg} / \mathrm{dl}$. Optimal glycaemic control was not associated with alcohol intake, smoking, obesity, central obesity and low physical activity levels.

Conclusions Most known diabetics had access to treatment but only approximately $25 \%$ were optimally treated. The need to optimally manage these patients is highlighted.

\section{P2-327 PHYSICAL ACTIVITY AND DEPRESSION IN ADOLESCENTS: CROSS-SECTIONAL RESULTS FROM THE ALSPAC COHORT}

doi:10.1136/jech.2011.142976k.59

${ }^{1} \mathrm{~N}$ Wiles, ${ }^{* 1} \mathrm{~A}$ Haase, ${ }^{1} \mathrm{D}$ Lawlor, ${ }^{1} \mathrm{~A}$ Ness, ${ }^{2} \mathrm{C}$ Riddoch, ${ }^{1} \mathrm{G}$ Lewis. ${ }^{1}$ University of Bristol, Bristol, UK; ${ }^{2}$ University of Bath, Bath, UK

Introduction Few studies have examined the association between physical activity (PA), measured objectively, and depressive symptoms in adolescents. The aim of this study was to determine whether there is an association between objective measures of PA (total PA and time spent in moderate and vigorous PA (MVPA)) and adolescent depressive symptoms.

Methods Data were available on 2452 adolescents aged 14 years participating in the ALSPAC cohort. Depressive symptoms were measured using the short version of the Moods and Feelings Questionnaire (MFQ) (self-report). Total PA (counts per minute (cpm)) and time spent in MVPA (minutes) were available based on accelerometry. The association between PA and MFQ scores was modelled using ordinal regression. 